\title{
The decreased expression of miR-429 in plasma exosomes after spinal cord injury inhibits neuronal apoptosis by mediating the PTEN/PI3K/Akt pathway
}

\author{
Jiayi Huang $^{1 \#}$, Chunshuai Wu ${ }^{1 \#}$, Guanhua $\mathrm{Xu}^{1}$, Yue Sun ${ }^{1}$, Chao Gui ${ }^{1}$, Jiawei Fu ${ }^{1}$, Zhiming Cui ${ }^{1}$, \\ Haiming Huang ${ }^{2}$
}

${ }^{1}$ Department of Spine Surgery, The Second Affiliated Hospital of Nantong University, Nantong University, Nantong, China; ${ }^{2}$ Department of Ultrasonography, The Second Affiliated Hospital of Nantong University, Nantong University, Nantong, China

Contributions: (I) Conception and design: J Huang, C Wu; (II) Administrative support: Z Cui, H Huang; (III) Provision of study materials or patients: G Xu; (IV) Collection and assembly of data: C Gui, J Fu; (V) Data analysis and interpretation: J Huang, Y Sun; (VI) Manuscript writing: All authors; (VII) Final approval of manuscript: All authors.

"These authors contributed equally to this work.

Correspondence to: Haiming Huang. Department of Ultrasonography, The Second Affiliated Hospital of Nantong University, Nantong University, Nantong 226001, China. Email: huanghaimingnt@163.com; Zhiming Cui. Department of Spine Surgery, The Second Affiliated Hospital of Nantong University, Nantong University, Nantong, China. Email: czmspine@163.com.

Background: Neuronal apoptosis after spinal cord injury (SCI) leads to sensorial and motorial dysfunction.
Exosomes are vesicles that contain many cellular components, including microRNA, and the role of miR-429
in plasma exosomes in this process after SCI requires further investigation.
Methods: The New York University impactor was used to create a rat model of SCI. We used SH-SY5Y
cells to construct a neuronal apoptotic cell model and extracted plasma exosomes from rats in a stimulation.
A miR-429 mimic and inhibitor were transfected, and the apoptosis-related indicators of the SH-SY5Y cells
were detected by using western blot, cell-counting kit-8 and immunofluorescence. The possible targets of
miR-429 were examined to verify the pathway of action. We then used the dual-luciferase reporter assay to
verify the binding of miR-429 with downstream molecules and speculate the mechanism of action.
Results: We successfully isolated and identified exosomes from plasma. Both the mean of adding
exosomes extracted from SCI-patients' plasma and knockdown of miR-429 in the culture of SH-SY5Y
cells promoted their apoptosis. Dual luciferase assays confirmed the interaction of miR-429 and 3'-
UTR region of phosphatase and tensin homolog (PTEN), which is the downstream target gene of miR-
429 , and the knockdown of miR-429 inhibits the phosphoinositide 3-kinase/protein kinase B pathway by
upregulating PTEN.

Conclusions: Our study showed that the decreased expression of miR-429 in SCI rat plasma exosomes promotes the apoptosis of nerve cells, which may be achieved by miR-429 interacting with PTEN and then affecting the PI3K/Akt pathway. This can be a possible mechanism of damage caused by SCI.

Keywords: Exosome; miR-429; phosphatase and tensin homolog (PTEN); apoptosis

Submitted Aug 16, 2021. Accepted for publication Jan 07, 2022.

doi: 10.21037/atm-21-5561

View this article at: https://dx.doi.org/10.21037/atm-21-5561 


\section{Introduction}

Spinal cord injury (SCI) is a major medical issue throughout the world and has very high rates of death and disability. The incidence of SCI has increased with the development of modern society, transportation, construction, and other industries, but there are few effective treatments for SCI $(1,2)$. SCI often results in irreversible sensorimotor impairment below the affected level $(1,2)$.

All prokaryotic and eukaryotic cells release extracellular vesicles (EVs) as part of their normal physiology and during pathologic events. EVs can be broadly divided into the following 2 categories: ectosomes and exosomes. Exosomes are vesicles that are $40-160 \mathrm{~nm}$ (average $100 \mathrm{~nm}$ ) in length and originate from the endosome (3). They contain many cellular components, including deoxyribonucleic acid (DNA), ribonucleic acid (RNA), lipids, metabolites, and cytoplasmic and cell surface proteins. Exosomes can pass through the blood-brain barrier and circulate in the peripheral blood to distant target organs, where they can be absorbed by nearby or distant cells, and subsequently modulate recipient cells (4). Common methods for identifying exosomes include WB for surface markers (CD63, TSG101), transmission electron microscopy (TEM) and nanoparticle tracking analysis for shape and particle size, and flow cytometry is also commonly used.

MicroRNAs (miRNAs) are small, single-stranded, noncoding RNAs with a length of 18-25 nucleotides. Their main mechanism of action is to inhibit protein translation by binding to or directly degrading specific mRNAs. Different tissues or cells express a large number of different miRNAs; these molecules are highly conserved with tissue specificity and play regulatory roles in different biological processes, such as growth and development, cell proliferation, differentiation, tumor proliferation, inflammation, and other physiological and pathological processes (5). miRNAs are involved in every aspect of the development and function of the nervous system under physiological conditions, including the maintenance of nerve cell morphology and structure, axon growth, and synaptic plasticity. Abnormal miRNA expression is associated with a variety of diseases and is highly conserved. As therapeutic targets, miRNAs have considerable advantages and good prospects in the development of new diagnostic and therapeutic methods and technologies for SCI.

MiRNAs are promising biomarkers; however, there is no unified standard for the clinical evaluation of SCI based on "free-circulating" miRNA profiles. This is likely because
miRNA analyses of biological fluids are affected by many pre-analysis variables. Conversely, exosomes are secreted by all cells and present in all biological fluids, and are attractive for the purposes of minimally invasive liquid biopsies with longitudinal sampling to track disease progression. The surface proteins on exosomes also promote their immune capture and enrichment. Exosome miRNAs are widely distributed in body fluids and have many advantages over free miRNAs. Specific miRNAs in the central nervous system can be transported via peripheral circulation and can be concentrated in exosomes. Ding et al. (6) characterized the miRNA profiles of serum exosomes in rats who underwent sham surgery and had acute SCI, and then analyzed the function and regulation of possible target genes using a comprehensive bioinformatics approach. The results showed that acute SCI induces changes in circulating exosome miRNA expression, and miR-429 was a significantly reduced miRNA. It has been confirmed that miR-429 can reverse the toxic effects of ketamine on PC12 cells by targeting BAG5 (7), and can also affect neuronal apoptosis by affecting the Wnt1 pathway (8). However, we did not find any literature on the effect of miR-429 in PLASMA exosomes of SCI on nerve cells.

The phosphoinositide 3-kinase/protein kinase B (PI3K/ $\mathrm{Akt}$ ) signaling pathway is an important intracellular signaling pathway that regulates the cell cycle. It is thus directly related to cell quiescence, proliferation, cancer, and longevity. Studies have found abnormal PI3K/Akt signaling in a variety of human cancers (e.g., gastric cancer, prostate cancer, and leukemia); this pathway can also affect chondrocyte autophagy and apoptosis in arthritis (9-12). Many studies have confirmed that the PI3K/AKT pathway plays a regulatory role in neurological diseases. The study of Liang et al. found that PI3K/AKT pathway can be involved in angiogenesis in the rat model of cerebral embolism (13). Activation of this pathway is also involved in neuroprotective effects in Alzheimer's disease (14). The pathway also plays a role in diseases related to spinal cord injury. For example, this pathway can affect pyroptosis of microglia after SCI (15) and interfere with microglia polarization after SCI (16). In addition, the PI3K/AKT pathway is also involved in a series of processes after SCI, including apoptosis, autophagy and vascular endothelial injury. As a well-known negative regulator of the PI3K/Akt pathway, phosphatase and tensin homolog (PTEN) can inhibit the phosphorylation and activation of Akt, thereby inhibiting pathway signaling (17). Studies have shown that miR-429 can affect PI3K/ Akt signaling by targeting PTEN, thereby inducing the 
occurrence of human non-small cell lung cancer and regulating liver cancer metastasis $(18,19)$. In this study, we assessed whether miR-429 and PTEN also play roles in exosome-mediated neuronal apoptosis after SCI.

We present the following article in accordance with the ARRIVE reporting checklist (available at https://dx.doi. org/10.21037/atm-21-5561).

\section{Methods}

\section{Establishment of a rat SCI model and specimen collection}

Experiments were performed under a project license (No. 20170222-001) granted by the Department of Animal Center, Nantong University, in compliance with Chinese national guidelines for the care and use of animals. The New York University impactor was used to create a rat model of SCI. Male Sprague Dawley rats aged 4 to 8 weeks and weighing 260 to $280 \mathrm{~g}$ were anesthetized and operated on in a prone position under aseptic conditions. The skin was cut longitudinally at the level of the thoracic 9 vertebral body, the spinous process of the vertebral body was extended, and the muscles were separated from each side, layer by layer, to expose the vertebral pedicle. The spinous process and pedicle of the thoracic 9 vertebral body were removed to expose the spinal cord. The thoracic 8 and 10 vertebral bodies were fixed with forceps, and a metal rod weighing $10 \mathrm{~g}$ was dropped from a height of $50 \mathrm{~mm}$ onto the exposed spinal cord surface, resulting in severe SCI. Afterward, the incision was sutured layer by layer, and the rats were returned to the cage. Rats in the control group were subjected to the surgery without the weight drop. Blood samples were collected from the tail vein of each animal 3 days after surgery. Each sample was placed in a sampling vessel, and centrifuged at $1,200 \mathrm{~g}$ at $4{ }^{\circ} \mathrm{C}$ for $10 \mathrm{~min}$. The supernatant (plasma) was carefully transferred into Eppendorf tubes and frozen at $-80{ }^{\circ} \mathrm{C}$ for subsequent experiments (20). A protocol was prepared before the study without registration.

\section{Exosome isolation and identification}

Exosomes were extracted from the rat plasma using Plasma Exosome Extraction Kits (Invitrogen, Waltham, MA, USA). In accordance with the manufacturer's instructions, the frozen plasma was removed from $-80{ }^{\circ} \mathrm{C}$, heated in a $37^{\circ} \mathrm{C}$ water bath for $10 \mathrm{~min}$, centrifuged at $10,000 \mathrm{rpm}$ for $20 \mathrm{~min}$, and the supernatant was then removed. Next, protease $\mathrm{K}$ inhibitor was added to the sample, which was heated in $37^{\circ} \mathrm{C}$ water bath for $10 \mathrm{~min}$. Next, the reagent was added for $30 \mathrm{~min}$, followed by centrifugation at 10,000 rpm for $5 \mathrm{~min}$, and the supernatant was discarded. The exosomes were resuspended in phosphate-buffer saline (PBS) and frozen at $-20{ }^{\circ} \mathrm{C}$ for subsequent experiments. The exosomes were identified by transmission electron microscopy (TEM), particle size and biomarkers.

\section{Cell cultures and identification}

SH-SY5Y cells were purchased from the National Collection of Authenticated Cell Cultures. In accordance with the conditions specified by the manufacturer, the cells were cultured in Dulbecco's minimum essential medium (DMEM) with high glucose (Gibco, Grand Island, NY, USA) supplemented with $10 \%$ fetal bovine serum (Gibco) in a constant temperature incubator at $37{ }^{\circ} \mathrm{C}$ under $5 \%$ carbon dioxide.

The cultured SH-SY5Y cells were prepared for immunofluorescence analyses. The cells were seeded in 24-well plates at a density of $10^{4}$ cells/well, and cultured for $24 \mathrm{~h}$. Once the cells reached $70 \%$ confluence, the culture medium was removed. The cells were then washed twice with phosphate buffered solution (PBS), fixed with $4 \%$ paraformaldehyde for $20 \mathrm{~min}$, and blocked with $1 \%$ bovine serum albumin for $30 \mathrm{~min}$. NeuN primary antibody (Proteintech Group, Rosemont, USA) was incubated overnight at $4{ }^{\circ} \mathrm{C}$ before incubation with a fluorescent secondary antibody. The nuclei were stained with $1 \mu \mathrm{g} / \mathrm{mL}$ of 6-diamino-2-phenylindole solution, and the cells were imaged under a fluorescence microscope (Olympus, Tokyo, Japan).

We stimulated SH-SY5Y cells with $400 \mu \mathrm{M}$ of hydrogen peroxide to construct a neuronal apoptosis model. Exosomes act as stimulants, and when the cells grow to $50 \%$ density, exosomes are added to the culture medium and co-cultured with the cells overnight.

\section{Western blotting}

The mature cells were lysed with radioimmunoprecipitation assay buffers. The proteins from the cells or exosomes were isolated by sodium dodecyl sulfate-polyacrylamide gel electrophoresis, and the isolated proteins were then transferred to polyvinylidene fluoride membranes. After blocking the membranes with $5 \%$ skim milk, they were incubated with primary antibodies against glyceraldehyde3-phosphate dehydrogenase, caspase-3 (Cas3), Bax, 
PTEN, PI3K, Akt, and phosphorylated (p)-Akt overnight at $4{ }^{\circ} \mathrm{C}$. The next morning, they were incubated at room temperature for $1 \mathrm{~h}$ with anti-mouse/rabbit secondary antibodies labeled with horseradish peroxidase, and enhanced chemiluminescence was used to develop the bands.

\section{Quantitative reverse transcription polymerase chain reaction ( $q R T-P C R)$}

Total RNA was extracted from the cells using TRIzol reagent (Invitrogen), and RNA concentration and purity were measured at 260 and $280 \mathrm{~nm}$ using an ultraviolet spectrophotometer. Total RNA was reverse transcribed into complementary DNA (cDNA) using a reverse transcription kit (Takara, Tokyo, Japan), and a qRT-PCR was performed using the Power SYBR Green Master Mix (Thermo Fisher Scientific, Waltham, MA, USA) on a quantitative PCR instrument (Thermo Fisher Scientific). The PCR protocol was as follows: $95^{\circ} \mathrm{C}$ for $10 \mathrm{~min}$, then $95{ }^{\circ} \mathrm{C}$ for $10 \mathrm{~s}+60^{\circ} \mathrm{C}$ for $30 \mathrm{~s}$ for 40 cycles. The results were analyzed using the $2^{-\Delta \Delta \mathrm{Ct}}$ relative expression method. Primer sequences used in this study were as follows: microRNA-429, F : 5 ' - GCCGATTAATA C T G TCTGGTAA - 3 ', R: 5 ' -CAGTGCAGGGTCCGAGGT-3 '; U6: F : 5 ' - C T C G C T T C G G C A G C A C A - 3 ', R : 5'-AACGCTTCACGAATTTGCGT-3'.

\section{Cell counting Kit 8 (CCK-8)}

The SH-SY5Y cells were inoculated into 96-well plates at a density of 3,000 cells per well. After the cells were firmly attached, the culture medium was removed, and the wells were washed twice with PBS. The exosome group was treated with medium containing $50 \mathrm{ng} / \mathrm{mL}$ exosomes from the SCI group/control group, and the positive control group was treated with complete medium containing $400 \mu \mathrm{M}$ hydrogen peroxide solution. After a continuous culture for $24 \mathrm{~h}, 10 \mu \mathrm{L}$ CCK-8 solution was added to each well, and the cells were incubated in a dark incubator for $1 \mathrm{~h}$. The absorbance at $450 \mathrm{~nm}$ was measured with a spectrophotometer (Bio-Rad, Hercules, CA, USA) to quantify the survival rate of the cells.

\section{MiRNA transfection}

The SY5Y cells were inoculated into 6-well plates and incubated with a miR-429 mimic, inhibitor, or negative control (NC) for $48 \mathrm{~h}$. All transfections were performed with a transfection kit (Ribo, Shanghai, China). After the cultures, the cells were collected for subsequent experiments.

\section{Dual-luciferase reporter assay system}

The wild-type PTEN 3'-UTR containing miR-429 complementary pairing sequence and mutant PTEN 3'-UTR were cloned into the pmirGLO (Invitrogen) to construct PTEN-WT-pmirGLO plasmid and PTEN-Mut-pmirGLO plasmid. HEK293T cells were cotransfected with miR-429 mimics (or NC mimics) and PTEN-WT (or PTEN-Mut) vectors. Transfection was conducted using Lipofectamine 3000 (Invitrogen). After $48 \mathrm{~h}$ of transfection, the luciferase activity was determined by a spectrophotometer (Bio-Rad). The relative luciferase activity was the ratio of firefly to Renilla luciferase activity.

\section{Statistical analysis}

All the data were analyzed using SPSS statistical software. The data are expressed as mean \pm standard deviation. Student $t$-tests were used for comparisons between two groups. A 1-way analysis of variance was used for comparisons among multiple groups. A $\mathrm{P}$ value $<0.05$ was considered statistically significant.

\section{Results}

\section{Identification of plasma exosomes}

The extracted exosomes were observed under TEM and were $~ 100$-nm spheres (see Figure 1A), which was consistent with the Nano-Sight particle size analysis results (see Figure $1 B, 1 C$ ), indicating that the extracted exosomes met the criteria and could be used for further experiments. Western bolt results also demonstrate the expression of exosomal surface specific markers (see Figure 1D).

\section{Neuronal marker expression in SH-SY5Y cells}

The SH-SY5Y cell line is derived from human neuroblastoma tissue and is often used as an experimental model to replace primary neurons. Immunofluorescence staining showed that SH-SY5Y cells expressed NeuN (see Figure 1E), which is a marker of mature neurons. 
A

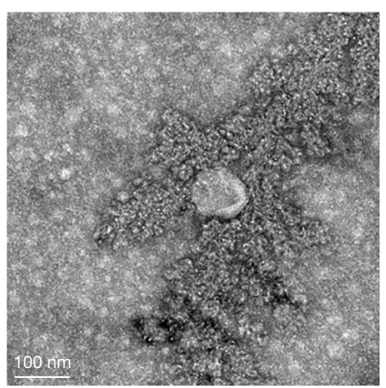

C

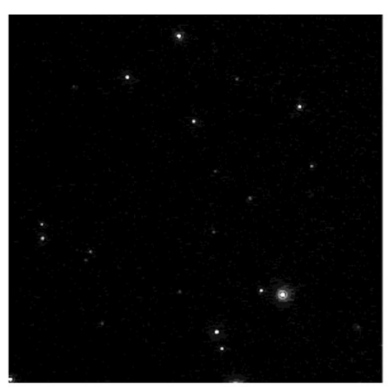

B

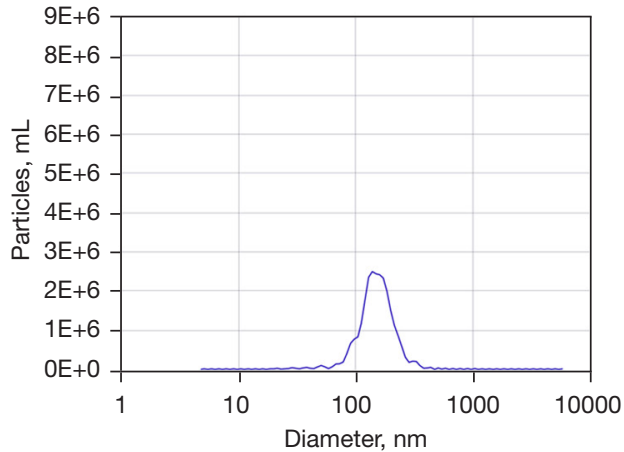

D

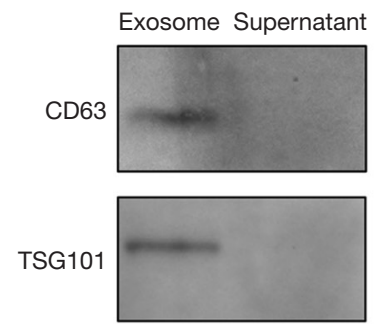

$\mathrm{E}$
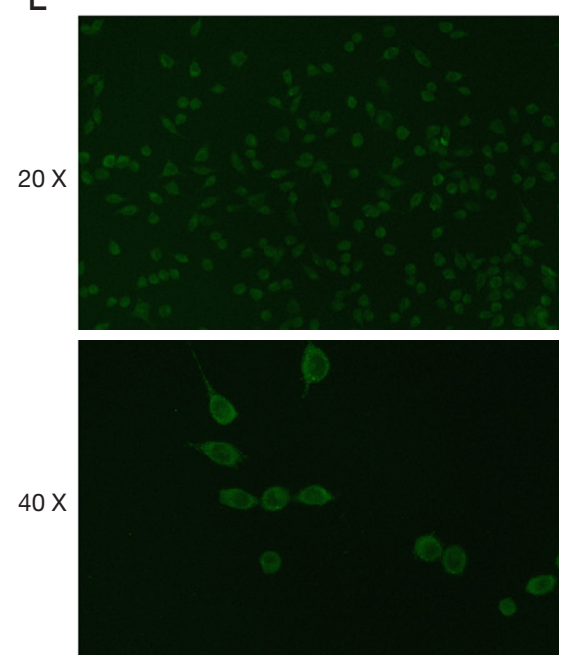
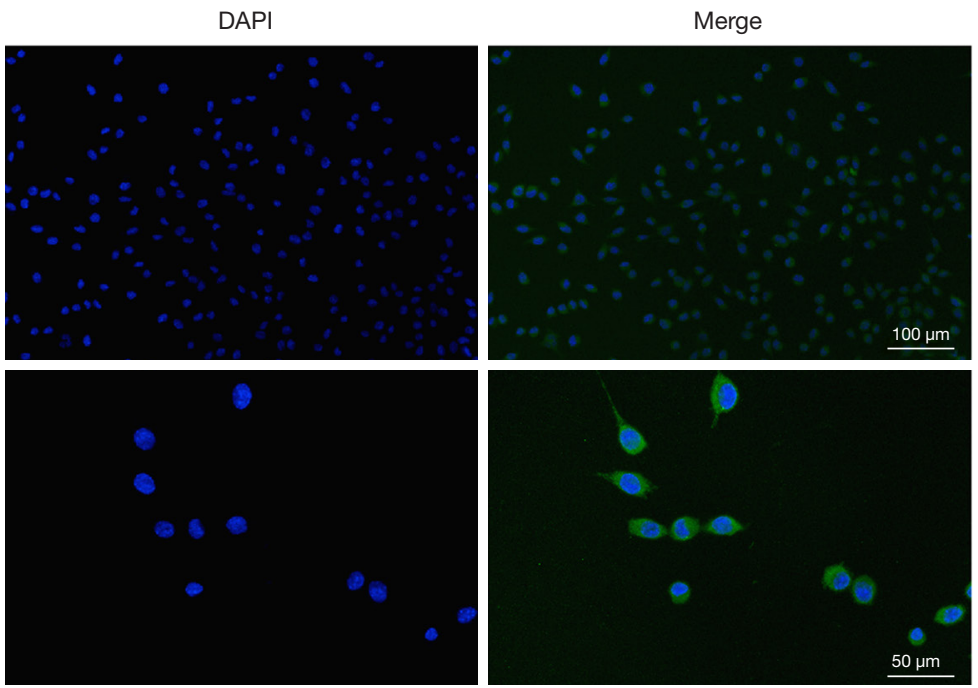

Figure 1 Identification of plasma-derived exosomes and SH-SY5Y cells. (A) TEM showing the size and morphology of the exosomes derived from the rat plasma; (B) the size and concentration of the plasma-derived exosomes by the NanosightNS300; (C) an image showing a snapshot of video tracking; (D) Western blot analysis of specific exosomal surface markers; (E) SH-SY5Y cells express NEUN, a marker of mature neurons. DAPI, 4',6-diamidino-2-phenylindole.

\section{SCI effect of plasma exosomes on SH-SY5Y cell apoptosis}

To explore the effect of SCI plasma exosomes on SH-SY5Y apoptosis after SCI, we detected the protein expression levels of the apoptosis-related molecules, such as Cas3 and Bax. The WB and immunofluorescence staining results showed that the protein levels of Cas3 and Bax were higher in $\mathrm{SH}$ -
SY5Y cells from the positive control group (adding $400 \mu \mathrm{M}$ hydrogen peroxide) and higher in the SCI group than the control group and NC group (see Figure 2A-2D). CCK-8 assays confirmed increased apoptosis in the positive control and SCI exosome groups (see Figure 2E). Collectively, these results indicate that plasma exosomes from SCI rats can promote $\mathrm{SH}-\mathrm{SY} 5 \mathrm{Y}$ cell apoptosis. 
A

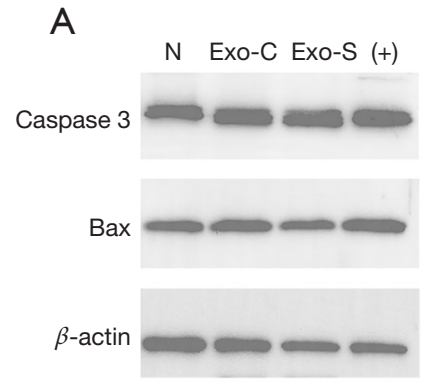

B

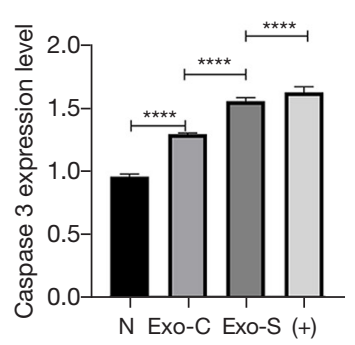

E
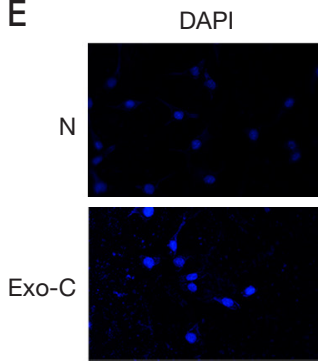
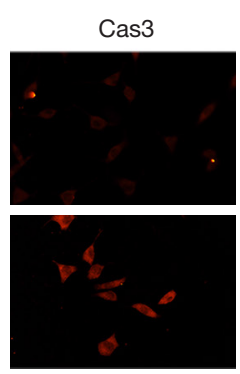

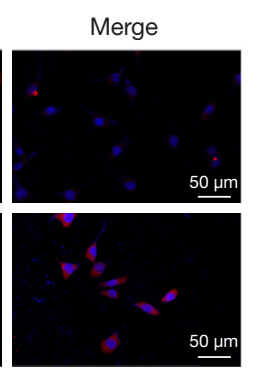

C

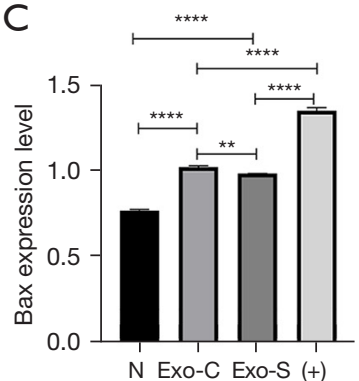

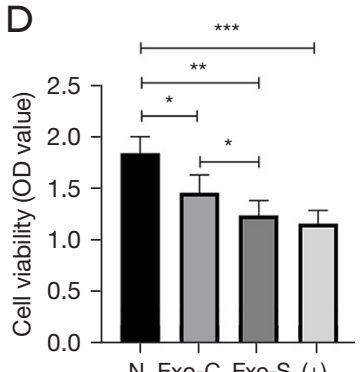

$\mathrm{N}$ Exo-C Exo-S (+)

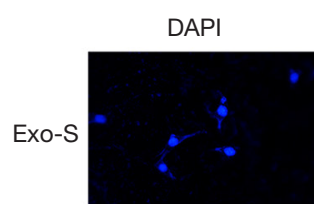

Cas3
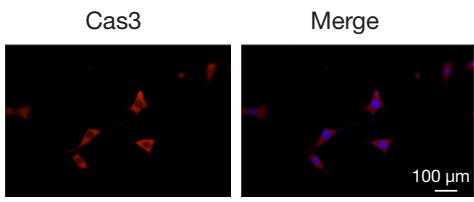

$(+)$
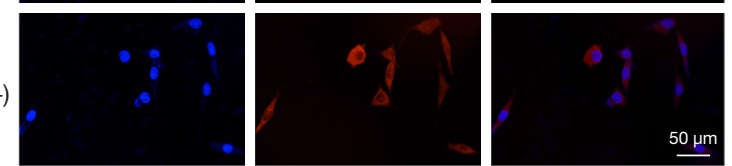

Figure 2 Exosomes extracted from SCI rats can promote apoptosis of SH-SY5Y cells. (A-C) Western blots were used to detect the expression of the apoptosis-related proteins Caspase 3 and Bax in SH-SY5Y cells stimulated by exosomes; (D) the survival rate of SHSY5Y cells under exosome stimulation was determined by CCK-8 assays; (E) the expression of caspase 3 in SH-SY5Y cells was detected by immunofluorescence. *, $\mathrm{P}<0.1$; ${ }^{* *}, \mathrm{P}<0.01$; ${ }^{* *}, \mathrm{P}<0.001$; **** $\mathrm{P}<0.0001$. Exo-C, Control Exosome; Exo-S, Spinal cord injury Exosome; DAPI, 4',6-diamidino-2-phenylindole; Cas3, caspase 3.

\section{MiR-130a knockout and overexpression affected SH-SY5Y cell apoptosis}

We transfected the cells with a miR-429 mimic and inhibitor to assess whether miR-429 promoted SY5Y cell apoptosis. The transfection efficiency of miR-429 was detected by qRT-PCR. After transfecting the miR429 mimic, miR-429 expression in the SY5Y cells was significantly higher than that in the NC group transfected with the miR-429 mimic. MiR-429 expression in the transfected miR-429 inhibitor group was lower than that in the inhibitor NC group, confirming successful transfection (see Figure 3A). The effects of miR-429 overexpression and knockout on SH-SY5Y cell apoptosis were also detected. The expression levels of apoptosis-related factors in the transfected miR-429 mimic group were lower than those in the NC mimic group, the apoptosis rate of the cells was significantly reduced (see Figure 3B), and apoptosis-related protein expression (i.e., of Cas 3 and Bax) was decreased (see Figures $3 C-3 E$ ). The levels were significantly higher in the miR-429 inhibitor group than the inhibitor NC group. The Cas3 immunofluorescence staining findings were consistent with the above results (see Figure 3F). Collectively, these data suggest that miR-429 overexpression and knockdown inhibits and promotes SH-SY5Y cell apoptosis, respectively.

\section{MiR-429 affects PI3K/Akt signaling by inbibiting PTEN}

MiRNAs regulate the expression of many genes at the posttranscriptional level. By searching the TargetScan, Starbase, miRanda and miRDB databases, we found that PTEN is a target of miR-429. To further demonstrate the interaction of miR-429 and 3'-UTR region of PTEN, the dual luciferase assays were performed. After co-transfection of miR-429 and PTEN-WT-pmirGLO, the luciferase activity was significantly decreased compared to the control $(\mathrm{P}<0.01)$ (see Figure 4A). As the PI3K/Akt pathway is involved in the regulation of cell proliferation and apoptosis, we studied the effects of miR-429 on the downstream targets of the PI3K/Akt signaling pathway. WBs were used to detect PTEN, PI3K, and p-Akt. The levels of all proteins were significantly lower in the miR-429 mimic group than the NC mimic group; however, the converse was found for the miR-429 inhibitor group (see Figure 4B-4D). These results were corroborated by the immunofluorescence results (see Figure 4E). Overall, the findings indicate that the 


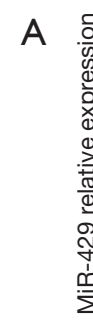

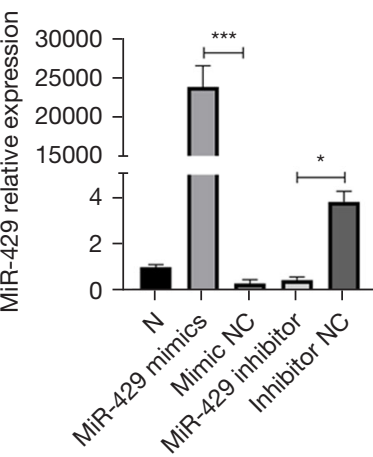

D

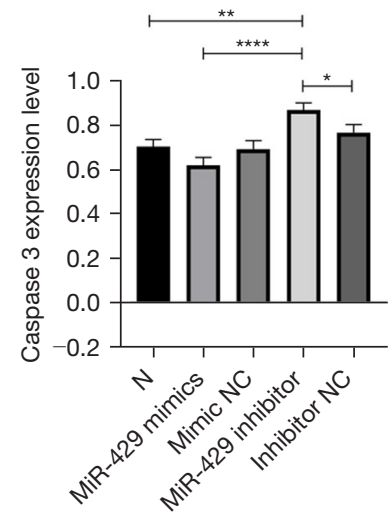

E

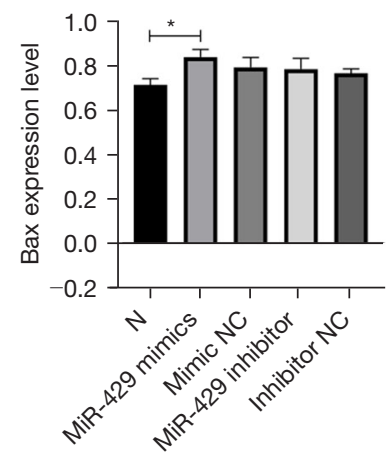

F
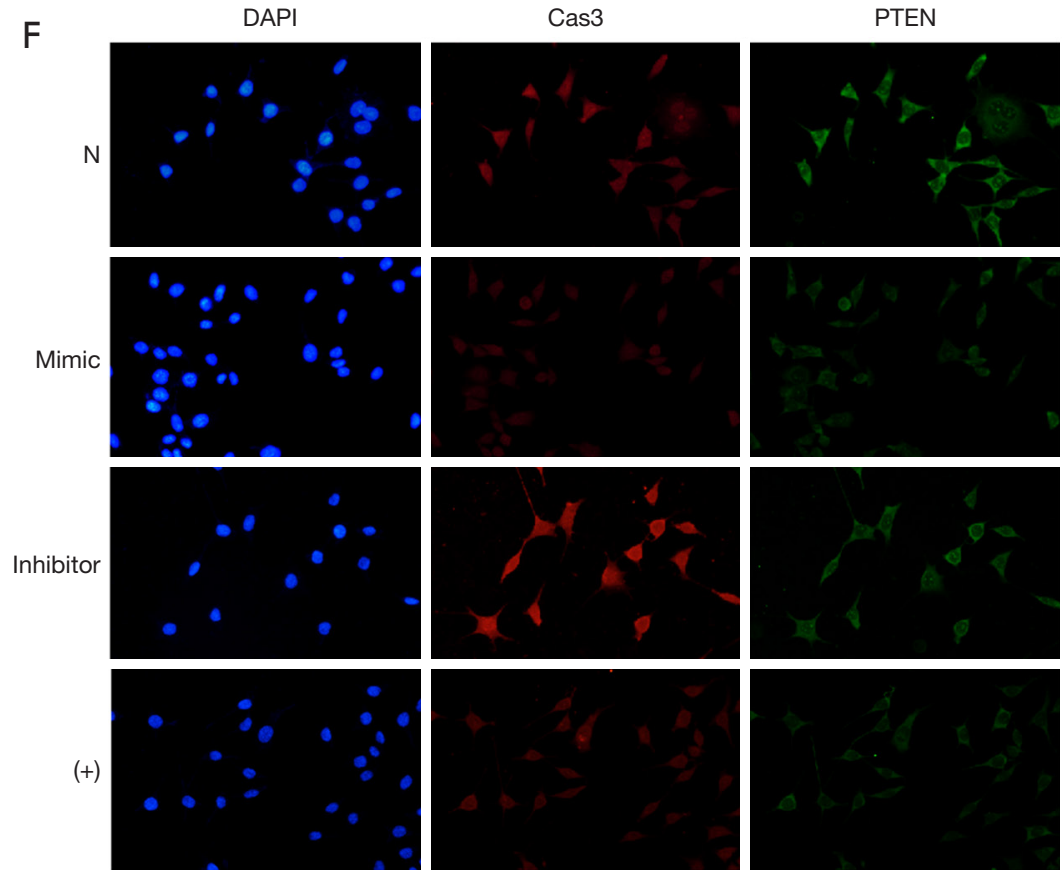

$(+)$
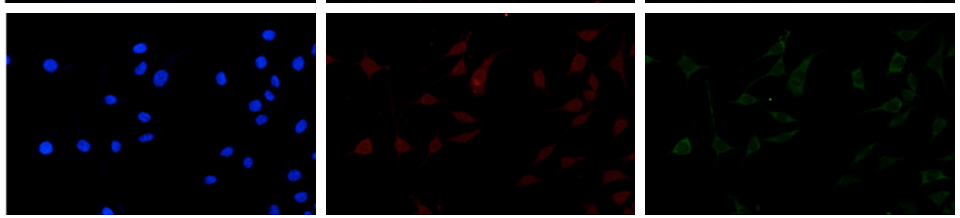

B

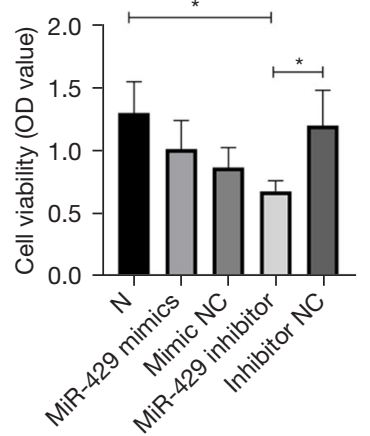

C
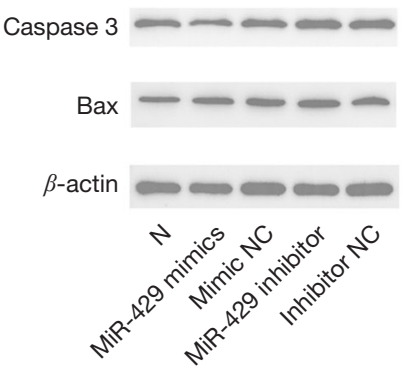
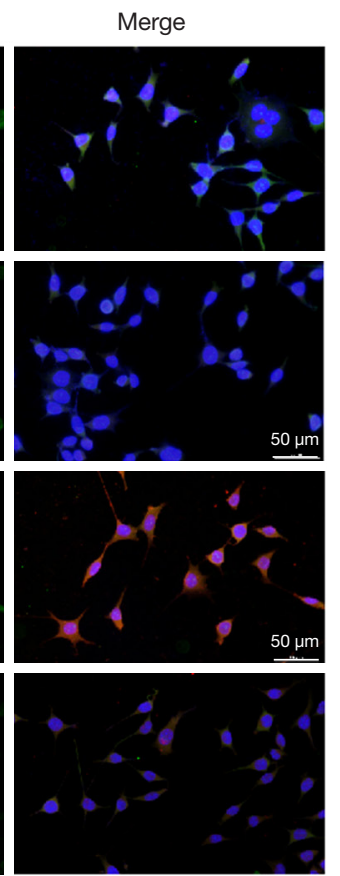

Figure 3 SH-SY5Y cells were transfected with a miR-429 mimic and inhibitor, and corresponding negative control microRNA. (A) The expression of miR-429 was detected by qRT-PCR; (B) the survival rate of SH-SY5Y cells was determined by CCK-8 assays; (C-E) Western blots were used to detect the expression of the apoptosis-related proteins Caspase 3 and Bax in SH-SY5Y cells; (F) the expression of Caspase 3 and PTEN in SH-SY5Y cells was detected by immunofluorescence. DAPI , 4',6-diamidino-2-phenylindole; Cas3, caspase 3. *, P<0.1; **, $\mathrm{P}<0.01 ;{ }^{* * *}, \mathrm{P}<0.001 ;{ }^{* * *}, \mathrm{P}<0.0001$. 
A

PTEN 3' UTR: 5' ...AAACUUUAUUUAUGGCAGUAUUC...

MiR-429: 3' UGCCAAAAUGGUCU--GUCAUAAU

B

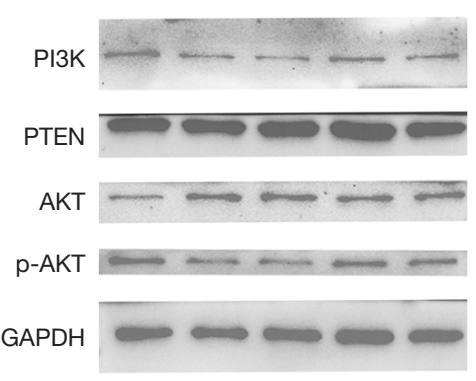

D

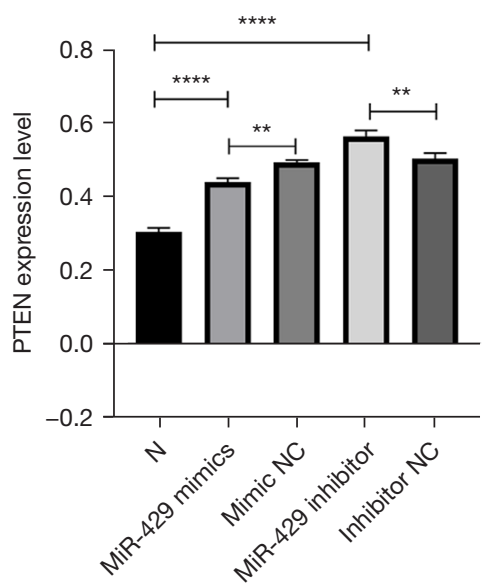

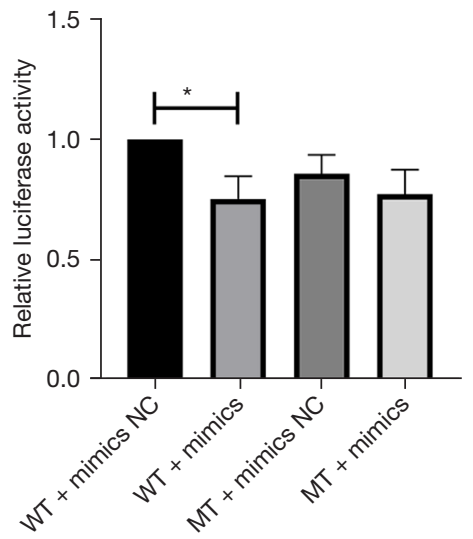

C

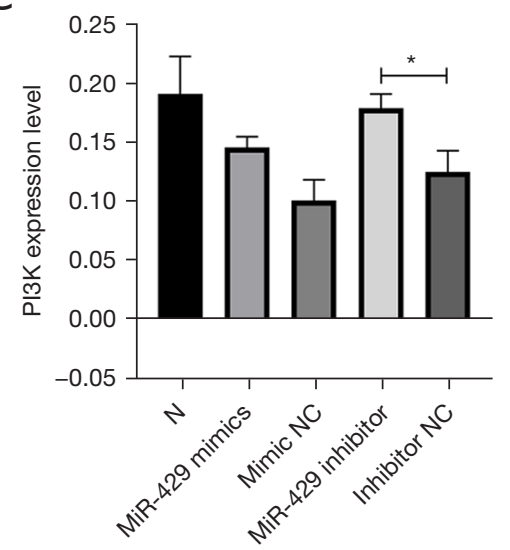

$\mathrm{E}$

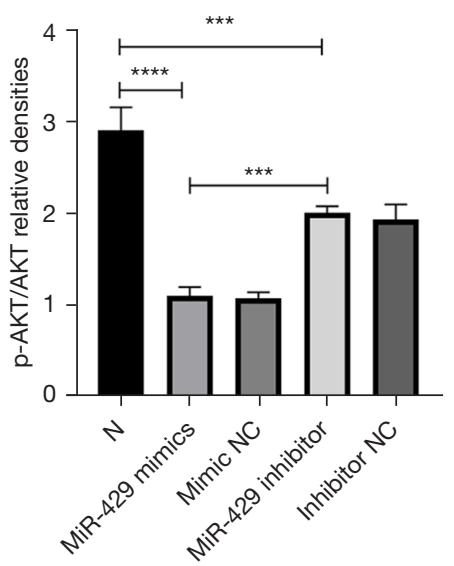

Figure 4 MiR-429 affects the PI3K/AKT pathway and the apoptosis of SH-SY5Y cells by targeting PTEN. (A) Binding regions between miR-429 and PTEN. The TargetScan, Starbase, miRanda, and miRDB databases were used to predict the putative target of miR-429. Dual luciferase assay was used to demonstrate the interaction of miR-429 and 3'-UTR region of PTEN. (B-E) Expression of PI3K/Akt pathway proteins after SH-SY5Y cells were transfected with a miR-429 mimic and inhibitor, and the corresponding NC microRNA. *, P<0.1; **, $\mathrm{P}<0.01 ;{ }^{* * *}, \mathrm{P}<0.001 ;{ }^{* * * *}, \mathrm{P}<0.0001$. 
overexpression of miR-429 inhibits SH-SY5Y cell apoptosis, and this effect may be mediated by the PTEN/Akt pathway.

\section{Discussion}

Neurons are terminal cells that cannot regenerate; thus it is critical to inhibit neuronal apoptosis after SCI. In this study, we compared the effects of plasma exosomes collected from SCI and control rats on neuronal apoptosis; miR-429 was selected as the study object after evaluating the sequencing results of plasma exosomes from SCI rats.

As an anti-apoptotic factor, the role of miR-429 in tumors has been widely recognized. miR-429 expression is significantly inhibited in gastric cancer (21) and colon cancer (22) and can inhibit thyroid cancer cell growth (23). In cervical cancer, miR-429 was shown to downregulate the expression of the inhibitor of nuclear factor (NF)- $\kappa \mathrm{B}$ $\mathrm{IkB}$, activate the NF- $\mathrm{\kappa B}$ pathway, reduce the production of interleukin-6 (IL-6) and interferon-B in cervical cancer cells, and ultimately inhibit cervical cancer development (24). As mentioned above, miR-429 is considered a tumor suppressor gene miRNA. However, a study showed that miR-429 overexpression inhibits renal cancer cell apoptosis, and its serum level is correlated with renal cancer patient prognosis (25). Thus, the effects of miR-429 on apoptosis remain questionable.

miR-429 is expressed at different levels in various cancers, and its functions also depend on the context. This variability may be due to the miRNA downregulation of many targets, including oncogenes and tumor suppressor genes. Yang et al. found that miR-429 inhibition reduced the inflammatory response in coronary heart disease by inhibiting the expression of inflammation-related factors IL- $1 \beta$, IL-6, and tumor necrosis factor- $\alpha$ (26), which is consistent with a report by $\mathrm{Lv}$ et al. that the downregulation of miR-429 reduced the lipopolysaccharide-induced pneumonia-induced response (27). In terms of the effect of miR-429 on neurons, Xiao et al. found that miR429 inhibition alleviated hypoxia-glucose deprivation/ reoxygenation-induced neuronal damage through GATAbinding protein 4 (28). Knocking down miR-429 may also mitigate amyloid $\beta$-induced cytotoxicity by targeting SOX2 and $\mathrm{Bcl} 2$ in mouse cortical neurons (29). However, there are few reports on how miR-429 affects cell growth or apoptosis through PTEN.

Inhibiting PTEN has been reported to increase the activation of the PI3K/Akt axis (17). Several recent studies have assessed the neuroprotective effect of PTEN inhibitors in the setting of cerebral ischemia injury. In an ischemic stroke model, PTEN was overexpressed and was shown to be involved in the development of ischemic stroke through the PI3K/Akt pathway (30). It was also reported that Bax, Caspase-3, and p53 are positively correlated with PI3K. As an inhibitor of the PI3K/Akt pathway, PTEN can significantly reduce p-Akt expression (31). miR-214 and other molecules have also been demonstrated to affect PI3K/Akt signaling through PTEN (e.g., by inhibiting neuronal apoptosis and promoting neuroblastoma cell proliferation) (32). PTEN is considered a target of miR429 , which explains the protective effect of miR-429 knockdown on neuronal apoptosis in SCI models.

Our study revealed that miR-429 overexpression and knockdown in SH-SY5Y cells inhibits and promotes apoptosis, respectively. Compared to the control group, the apoptosis rate was decreased in SH-SY5Y cells transfected with the miR-429 mimic. WB further showed a lower expression of apoptosis-related proteins (Cas3 and Bax/ $\mathrm{Bcl} 2)$ in the miR-429 mimics group. The opposite was true for the miR-429 inhibitor group. miR-429 may be one of the mechanisms of exosome-mediated nerve injury after SCI, and could be a possible pathway for the treatment of nerve injuries.

This study had several limitations. Firs, SH-SY5Y cells were used to replace primary neurons. This cell line is derived from human neuroblastoma tissue, and many studies have used all-trans retinoic acid (RA) to induce differentiation to obtain more neuron-like characteristics, including axonal growth and morphological changes (33). Undifferentiated SH-SY5Y cells have also been used as a model line $(34,35)$, and one report showed no significant difference in neuronal properties between undifferentiated SK-N-SH cells (a sister line of SH-SY5Y cells) and RAdifferentiated SK-N-SH cells (36). However, the need for RA-induced differentiation remains controversial. Our findings might be more physiologically relevant if they were confirmed in primary neurons. Further, the mammalian target protein of rapamycin (mTOR) is an important downstream protein of PI3K/Akt signaling and a major regulator of this pathway (37). We detected the expression of p-Akt; however, we did not assess downstream mTOR. Finally, while the PI3K/Akt pathway is an important signaling pathway affecting cell survival and apoptosis, we did not conduct salvage experiments to examine the role of PTEN. These issues should be addressed in future 
investigations.

\section{Conclusions}

The results of this study indicate that low miR-429 expression in SCI plasma exosomes promotes neuron apoptosis by interacting with PTEN and affecting PI3K/ Akt signaling.

\section{Acknowledgments}

Funding: This work was supported by Natural Science Foundation of China (81771319); Nantong City Science and Technology Project (MS12019027, HS2018002 and MA2021016); Medical Research Project of Jiangsu Commission of Health (ZDB2020004).

\section{Footnote}

Reporting Checklist: The authors have completed the ARRIVE reporting checklist. Available at https://dx.doi. org/10.21037/atm-21-5561

Data Sharing Statement: Available at https://dx.doi. org/10.21037/atm-21-5561

Conflicts of Interest: All authors have completed the ICMJE uniform disclosure form (available at https://dx.doi. org/10.21037/atm-21-5561). The authors have no conflicts of interest to declare.

Ethical Statement: The authors are accountable for all aspects of the work in ensuring that questions related to the accuracy or integrity of any part of the work are appropriately investigated and resolved. Experiments were performed under a project license (No. 20170222-001) granted by the Department of Animal Center, Nantong University, in compliance with Chinese national guidelines for the care and use of animals.

Open Access Statement: This is an Open Access article distributed in accordance with the Creative Commons Attribution-NonCommercial-NoDerivs 4.0 International License (CC BY-NC-ND 4.0), which permits the noncommercial replication and distribution of the article with the strict proviso that no changes or edits are made and the original work is properly cited (including links to both the formal publication through the relevant DOI and the license).
See: https://creativecommons.org/licenses/by-nc-nd/4.0/.

\section{References}

1. Alizadeh A, Dyck SM, Karimi-Abdolrezaee S. Traumatic Spinal Cord Injury: An Overview of Pathophysiology, Models and Acute Injury Mechanisms. Front Neurol 2019;10:282.

2. Shank CD, Walters BC, Hadley MN. Current Topics in the Management of Acute Traumatic Spinal Cord Injury. Neurocrit Care 2019;30:261-71.

3. Kalluri R, LeBleu VS. The biology, function, and biomedical applications of exosomes. Science 2020;367:eaau6977.

4. Zhang J, Li S, Li L, et al. Exosome and exosomal microRNA: trafficking, sorting, and function. Genomics Proteomics Bioinformatics 2015;13:17-24.

5. Tigchelaar S, Gupta R, Shannon CP, et al. MicroRNA Biomarkers in Cerebrospinal Fluid and Serum Reflect Injury Severity in Human Acute Traumatic Spinal Cord Injury. J Neurotrauma 2019;36:2358-71.

6. Ding SQ, Chen J, Wang SN, et al. Identification of serum exosomal microRNAs in acute spinal cord injured rats. Exp Biol Med (Maywood) 2019;244:1149-61.

7. Fan X, Bian W, Liu M, et al. MiRNA-429 alleviates ketamine-induced neurotoxicity through targeting BAG5. Environ Toxicol 2021;36:620-7.

8. Fang H, Li HF, He MH, et al. Long non-coding RNA MALAT1 sponges microRNA-429 to regulate apoptosis of hippocampal neurons in hypoxic-ischemic brain damage by regulating WNT1. Brain Res Bull 2019;152:1-10.

9. Fruman DA, Chiu H, Hopkins BD, et al. The PI3K Pathway in Human Disease. Cell 2017;170:605-35.

10. Bertacchini J, Heidari N, Mediani L, et al. Targeting $\mathrm{PI} 3 \mathrm{~K} / \mathrm{AKT} / \mathrm{mTOR}$ network for treatment of leukemia. Cell Mol Life Sci 2015;72:23 37-47.

11. Chen H, Zhou L, Wu X, et al. The PI3K/AKT pathway in the pathogenesis of prostate cancer. Front Biosci (Landmark Ed) 2016;21:1084-91.

12. Verret B, Cortes J, Bachelot T, et al. Efficacy of PI3K inhibitors in advanced breast cancer. Ann Oncol 2019;30:x12-20.

13. Liang Z, Chi YJ, Lin GQ, et al. MiRNA-26a promotes angiogenesis in a rat model of cerebral infarction via PI3K/ AKT and MAPK/ERK pathway. Eur Rev Med Pharmacol Sci 2018;22:3485-92.

14. Li L, Xu Y, Zhao M, et al. Neuro-protective roles of long non-coding RNA MALAT1 in Alzheimer's disease with the involvement of the microRNA-30b/CNR1 network 
and the following PI3K/AKT activation. Exp Mol Pathol 2020;117:104545.

15. Xu S, Wang J, Zhong J, et al. CD73 alleviates GSDMDmediated microglia pyroptosis in spinal cord injury through PI3K/AKT/Foxo1 signaling. Clin Transl Med 2021;11:e269.

16. Liu W, Rong Y, Wang J, et al. Exosome-shuttled miR216a-5p from hypoxic preconditioned mesenchymal stem cells repair traumatic spinal cord injury by shifting microglial M1/M2 polarization. J Neuroinflammation 2020;17:47.

17. Worby CA, Dixon JE. PTEN. Annu Rev Biochem 2014;83:641-69.

18. Lang Y, Xu S, Ma J, et al. MicroRNA-429 induces tumorigenesis of human non-small cell lung cancer cells and targets multiple tumor suppressor genes. Biochem Biophys Res Commun 2014;450:154-9.

19. Tang J, Li L, Huang W, et al. MiR-429 increases the metastatic capability of $\mathrm{HCC}$ via regulating classic Wnt pathway rather than epithelial-mesenchymal transition. Cancer Lett 2015;364:33-43.

20. Wu C, Bao G, Xu G, et al. Triad1 regulates the expression and distribution of EHD1 contributing to the neurite outgrowth of neurons after spinal cord injury. J Cell Biochem 2019;120:5355-66.

21. Zhang M, Dong BB, Lu M, et al. miR-429 functions as a tumor suppressor by targeting FSCN1 in gastric cancer cells. Onco Targets Ther 2016;9:1123-33.

22. Tian X, Wei Z, Wang J, et al. MicroRNA-429 inhibits the migration and invasion of colon cancer cells by targeting PAK6/cofilin signaling. Oncol Rep 2015;34:707-14.

23. Wu G, Zheng H, Xu J, et al. miR-429 suppresses cell growth and induces apoptosis of human thyroid cancer cell by targeting ZEB1. Artif Cells Nanomed Biotechnol 2019;47:548-54.

24. Fan JY, Fan YJ, Wang XL, et al. miR-429 is involved in regulation of $\mathrm{NF}-\kappa$ Bactivity by targeting IKK $\beta$ and suppresses oncogenic activity in cervical cancer cells. FEBS Lett 2017;591:118-28.

25. Wang ZK, Luo L, Du ZJ, et al. MiR429 expression level in renal cell cancer and its correlation with the prognosis of patients. J BUON 2017;22:1428-33.

26. Yang Q, Li J, Zhang H, et al. Down-regulation of microRNA-429 alleviates myocardial injury of rats with coronary heart disease. Cell Cycle 2019;18:2550-65.

27. Lv H, Zhang S, Hao X. Swainsonine protects H9c2 cells against lipopolysaccharide-induced apoptosis and inflammatory injury via down-regulating miR-429. Cell
Cycle 2020;19:207-17.

28. Xiao J, Kong R, Hu J. Inhibition of microRNA-429 attenuates oxygen-glucose deprivation/reoxygenationinduced neuronal injury by promoting expression of GATA-binding protein 4. Neuroreport 2018;29:723-30.

29. Fu S, Zhang J, Zhang S. Knockdown of miR-429 Attenuates A $\beta$-Induced Neuronal Damage by Targeting SOX2 and BCL2 in Mouse Cortical Neurons. Neurochem Res 2018;43:2240-51.

30. Zheng T, Shi Y, Zhang J, et al. MiR-130a exerts neuroprotective effects against ischemic stroke through PTEN/PI3K/AKT pathway. Biomed Pharmacother 2019;117:109117.

31. Nie ZY, Yang L, Liu XJ, et al. Morin Inhibits Proliferation and Induces Apoptosis by Modulating the miR-188-5p/ PTEN/AKT Regulatory Pathway in CML Cells. Mol Cancer Ther 2019;18:2296-307.

32. Wu Q, Shang Y, Shen T, et al. Neuroprotection of miR214 against isoflurane-induced neurotoxicity involves the PTEN/PI3K/Akt pathway in human neuroblastoma cell line SH-SY5Y. Arch Biochem Biophys 2019;678:108181.

33. Påhlman S, Ruusala AI, Abrahamsson L, et al. Retinoic acid-induced differentiation of cultured human neuroblastoma cells: a comparison with phorbolesterinduced differentiation. Cell Differ 1984;14:135-44.

34. Levites Y, Amit T, Youdim MB, et al. Involvement of protein kinase $\mathrm{C}$ activation and cell survival/ cell cycle genes in green tea polyphenol (-)-epigallocatechin 3-gallate neuroprotective action. J Biol Chem 2002;277:30574-80.

35. Levites Y, Youdim MB, Maor G, et al. Attenuation of 6-hydroxydopamine (6-OHDA)-induced nuclear factorkappaB (NF-kappaB) activation and cell death by tea extracts in neuronal cultures. Biochem Pharmacol 2002;63:21-9.

36. Lombet A, Zujovic V, Kandouz M, et al. Resistance to induced apoptosis in the human neuroblastoma cell line SK-N-SH in relation to neuronal differentiation. Role of Bcl-2 protein family. Eur J Biochem 2001;268:1352-62.

37. Murugan AK. mTOR: Role in cancer, metastasis and drug resistance. Semin Cancer Biol 2019;59:92-111.

(English Language Editor: L. Huleatt)

Cite this article as: Huang J, Wu C, Xu G, Sun Y, Gui C, Fu J, Cui Z, Huang H. The decreased expression of miR-429 in plasma exosomes after spinal cord injury inhibits neuronal apoptosis by mediating the PTEN/PI3K/Akt pathway. Ann Transl Med 2022;10(1):6. doi: 10.21037/atm-21-5561 